\title{
Trends in Airline Passenger Trip Delays (2007): A Multi-Segment Itinerary Analysis
}

\author{
Lance Sherry (Ph.D) ${ }^{1}$, Ashwin Samant (Ph.D. Candidate) $)^{2}$, Guillermo Calderon-Meza (Ph.D. Candidate) ${ }^{3}$ \\ Center for Air Transportation Systems Research, George Mason University, Fairfax, VA, 22031
}

\begin{abstract}
The raison d'etre of the Airline Passenger Transportation System (APTS) is the rapid, affordable, and safe transportation of passengers (and cargo). Traditional APTS performance metrics, such as flight delays and cancellation rates, fail to account for the delays to passenger trips caused by cancelled flights, missed connections and diversions. Previous research estimated Passenger Trip Delays statistics for single segment passenger itineraries (i.e. direct itineraries) only. This paper describes the results of analysis of the Passenger Trip Delays for multi-segment itineraries (i.e. direct and connecting itineraries). The results provide a nuanced view of the performance of the APTS, the causes of passenger trip delays, and mitigation strategies.

In 2007, 6.8M flights provided service to 299 airports. The network of scheduled flights supported an estimated 51M passenger itineraries. Thirteen percent of the itineraries provided direct service, $87 \%$ provided connecting service. Direct itineraries carried an average of 30 passengers, while connecting itineraries carried an average of 4 passengers, resulting in an even split between passengers on direct and connecting itineraries. In terms of performance, $31 \%$ of the itineraries were disrupted yielding passenger trip delays totaling 15,841 years. Forty eight percent of the total trip delays were the result of itineraries disrupted by delayed flights, $31 \%$ by cancelled flights, $19 \%$ by missed connections, and 25\% by diverted flights. Connecting itineraries, through hubs or not, were more likely to experience disruptions. The asymmetries in type of itinerary, load factor, and type of disruption provide insights into the inherent structure of the system and explain why a single performance metric cannot incentivize uniform performance improvement. The implications for NextGen and air transportation policy are discussed.
\end{abstract}

\section{Introduction}

The Airline Passenger Transportation System (APTS) provides rapid, affordable, and safe transportation services to passengers and cargo. The affordability of this mode of transportation is determined by the airfare as well as additional costs (i.e. parking, food, local transportation, taxes, and fees). Poor reliability of the transportation service generates additional costs including lost productivity [1], unplanned ground transportation connections, unexpected overnight stays, as well as "trip delay mitigation measures" such as leaving a day early or returning a day late [2]. The cost of lost productivity and trip delay to passengers in 2007 is estimated to result in an annual loss to the U.S. economy of \$16.1B [2].

Traditional measures of airline transportation system performance focus on flight delays and flight cancellation rates. These metrics fail to account for the trip delays experienced by passengers due to cancelled flights, missed connections, and diverted flights.

Algorithms for estimating passenger trip delays have evolved from a single airline hub itinerary analysis using one month of proprietary airline data developed by Bratu \& Barnhart ${ }^{3}$, to all airline single segment flight (i.e. direct itineraries only) using publicly available data developed by Wang \& Sherry ${ }^{4,5}$, to all airline single segment flights (i.e. direct itineraries only) with connecting rebooking strategies and diverted flight routes using publicly available data developed by Sherry \& Calderon-Meza ${ }^{6} \mathrm{Zhu}^{7}$. This paper describes the results of analysis of passenger trip delays using publicly available data from the Bureau of Transportation Statistics [8] and an algorithm that generates multi-segment itineraries (i.e. direct as well as connecting itineraries).

The results of analysis of 2007 data provide a nuanced view of the performance of the airline passenger transportation system useful for planning for NextGen and developing consumer protection policies. The highlights of the analysis are listed below:

- In 2007, 6.9M flights provided service to 299 airports. These flights were networked to provide 51M passenger itineraries. A total of $388 \mathrm{M}$ passengers were transported.

\footnotetext{
${ }^{1}$ Director, Center for Air Transportation Systems Research, System Engineering and Operations Research Dept, George Mason University, 4400 University Drive, MS4A6, Fairfax, VA, 22031. AIAA Member

${ }^{22}$ Center for Air Transportation Systems Research, George Mason University, 4400 University Drive, MS:4A6, Fairfax, VA, 22031

${ }^{3}$ Center for Air Transportation Systems Research, George Mason University, 4400 University Drive, MS:4A6, Fairfax, VA, 22031
} 
- The ratio of direct itineraries (13\%) to connecting itineraries (87\%) was 1:6.8. The ratio between passengers per itinerary for direct itineraries (average 29.9 passengers per itinerary) and connecting itineraries (average 4.3 passengers per itinerary) was $7: 1$. The asymmetry between type of itineraries (1:6.8) and passengers per itinerary (7:1) resulted in an even split between total passengers on direct (196K passengers) and connecting (190K passengers) itineraries.

- Itineraries disrupted by delayed, cancelled or diverted flights, or by missed connections, yielded a total of 15,841 years of trip delays. Forty-eight percent of total delays were due to delayed flights, 19\% due to missed connections, $31 \%$ due to cancelled flights, and $2 \%$ due to diverted flights.

- Twenty-nine percent of the passenger trips were disrupted (i.e. approximately one out of three). Twenty five percent of the disruptions were due to delayed flights, $2 \%$ due to cancelled flights, $1.2 \%$ due to missed connections, and $0.2 \%$ due to diverted flights.

- The average passenger trip delay was 22 minutes. However for passengers that experienced trip disruptions, their average trip delay was 72 minutes. The average delay for passengers by type of itinerary disruption was; 40 minutes for passengers on itineraries with delayed flights, 5 hours and 24 minutes for passengers on itineraries with cancelled flights, 5 hours and 46 minutes for passengers with missed connection itineraries, and 4 hours and 17 minutes for passengers on itineraries with diverted flights.

- Passengers on direct itineraries $(25 \%)$ were less likely to experience disrupted itineraries, than passengers on connecting itineraries $(32 \%)$.

These results indicate the overall brittle nature of the ATPS. A passenger has between a one-in-three chance and a onein-four chance of experiencing a trip disruption. These disruptions on average are for 72 minutes, but can result in overnight stays (e.g. passenger cannot be rebooked on the same day when a fight is cancelled or a connection is missed).

These results also provide insight into the inherent structure of how passengers are transported via passenger itineraries. In 2007, the network of flights included 7 times more connecting itineraries than direct itineraries, with an equal number of passengers on direct and connecting itineraries. The increased likelihood of itinerary disruptions on connecting itineraries. suggests that airlines were better able to protect passengers on direct itineraries (even those on flights to the hubs) than on complex connecting itineraries. For example, connecting itineraries were 21 times more likely to be disrupted by cancelled flights than direct itineraries.

A new perspective that has come out of these results is that it may not be possible to create a single "one-size-fits-all" performance metric to incentivize satisfactory consumer protection standards. The differences between itineraries and the complexities in managing each type of itinerary require a different approach.

This paper is organized as follows. Section II describes the structure and the dynamics of the air transportation system. Section III describes the data and algorithms used to derive the passenger itineraries and compute the passenger trip delays. Section IV provides the results of the analysis for 2007. Section V discusses the implications of these results.

\section{Structure and Dynamics of the Airline Passenger Transportation System (APTS)}

The APTS transports passengers on flights $\left(f_{i} \in F\right)$ between their Origins and their Destinations. A flight is defined by a date, a flight number, an origin/destination, a scheduled departure time, a scheduled arrival time, an actual departure time, and an actual arrival a time, available seats, load factor, and a flight status: on-time, delayed, cancelled, diverted.

Airlines schedule flights to operate in a time-space network of flights $\left(\mathrm{n}_{\mathrm{i}} \epsilon \mathrm{N}\right)$ such that aircraft and crews can be positioned to operate the flights in contiguous manner throughout the day. The flights are also scheduled to enable passengers to connect from one flight to the next. Feasible sequences of flights to ferry passengers from an Origin to a Destination are known as passenger itineraries $\left(\mathrm{p}_{\mathrm{i}} \in \mathrm{PI}\right)$. A passenger itinerary is defined as one flight (e.g. $\left.\mathrm{f}_{103}\right)$ or a sequence of flights $\left(\mathrm{f}_{46}\right.$, $f_{93}$, along with the number of passengers on this itinerary. For the purpose of this study, a feasible sequence for connecting flights includes a scheduled connecting window of more than 30 minutes and less than 3 hours. A passenger itinerary is classified by an itinerary status: on-time, delayed, rebooked due to missed connection, rebooked due to cancellation, and diverted.

By definition, a flight $\left(f_{i}\right)$ will have passengers from one or more passenger itineraries $\left(p_{i}, p_{j}, p_{k} \ldots\right)$. For example a Delta flight from Washington, D.C. to Atlanta, will have passengers flying from DCA to ATL, as well as passengers flying from DCA to ATL and connecting at ATL to other destinations. The passengers on a given flight $\left(f_{i}\right)$ will be the sum of all the passengers on each passenger itineraries $\left(\mathrm{p}_{\mathrm{i}}\right)$ that form that flight. 


\begin{tabular}{|l|l|l|l|}
\hline Passenger Itinerary Status & Direct Itinerary (O-D) & Connecting Itinerary (O-H-D) & $\begin{array}{l}\text { Passenger Trip Delay is a } \\
\text { function of .. }\end{array}$ \\
\hline On-time & O-D flight on-time & $\begin{array}{l}\text { O-H flight on-time or delayed or } \\
\text { diverted (but within connection } \\
\text { time), and H-D flight on-time }\end{array}$ & N/A \\
\hline Delayed & O-D flight: delayed & $\begin{array}{l}\text { O-H flight on-time or delayed or } \\
\text { diverted (but within connection } \\
\text { time), and H-D flight on-time }\end{array}$ & Magnitude of flight delay \\
\hline $\begin{array}{l}\text { Passenger rebooked due to } \\
\text { Missed Connection }\end{array}$ & N/A & $\begin{array}{l}\text { O-H flight delayed or diverted } \\
\text { and not within connection time, } \\
\text { and rebooked H-D flight on- } \\
\text { time, delayed, or diverted }\end{array}$ & $\begin{array}{l}\text { Availability of seats on later } \\
\text { flights and frequency of } \\
\text { flights from H-D }\end{array}$ \\
\hline $\begin{array}{l}\text { Passenger rebooked due to } \\
\text { cancelled flights }\end{array}$ & $\begin{array}{l}\text { Rebooked O-D flight } \\
\text { on-time, delayed, or } \\
\text { diverted }\end{array}$ & $\begin{array}{l}\text { O-H flight cancelled, and } \\
\text { rebooked O-D flight or O-H-D } \\
\text { flight on-time, delayed, or } \\
\text { diverted }\end{array}$ & $\begin{array}{l}\text { Availability of seats on later } \\
\text { flights and frequency of } \\
\text { flights from O-D, O-H-D, } \\
\text { and O-H1-D }\end{array}$ \\
\cline { 2 - 4 } & $\begin{array}{l}\text { O-H flight on-time, delayed, } \\
\text { diverted (but within connection } \\
\text { time), and H-D flight cancelled } \\
\text { and rebooked }\end{array}$ & $\begin{array}{l}\text { Magnitude of delay due to } \\
\text { diversion }\end{array}$ \\
\hline Diverted & O-D flight diverted & \begin{tabular}{l} 
H-D flight diverted \\
\hline
\end{tabular}
\end{tabular}

The relationship between flight disruptions and passenger itinerary disruptions. Also describes the characteristics of the passenger trip delays.

Table 1

When a flight is disrupted (i.e. delayed, cancelled, or diverted), the associated passenger itineraries maybe disrupted. The relationship between a flight disruption and a passenger itinerary disruption is summarized in Table 1 (columns 1-3). The magnitude of the delay is also summarized in Table 1 (column 4). For passenger itineraries affected by flight disruptions only (e.g. delayed and diverted flights), the passenger trip delay experienced by passengers on these itineraries is determined by the magnitude of the flight delay. For passenger itineraries disrupted by cancelled flights or missed connections, the passenger trip delay experienced by these passengers is determined by the frequency of flights to the destination later in the day and the availability of seats on these flights. The available seats for rebooking passengers for a given flight is known as the "seat reservoir."

This distinction is important for consumer protection and modernization benefits assessment. Reducing flight delays yields direct benefits to the itineraries disrupted by delayed flights, but not to itineraries disrupted by cancelled flights or missed connections. Cancelled flights and missed connections constitute 50\% of the total passenger trip delays (see Results section below).

The following list provides a summary of terms and identities used in this paper.

- A flight transports passengers from an origin to a destination

- A passenger itinerary defines how passengers are transported from origin to destination

- A direct passenger itinerary transports passengers with a single flight

- A connecting passenger itinerary transports passengers with two (or more) flights

- Passengers on a flight $=\sum$ passengers on all passenger itineraries that include this flight

- The Total Passengers Transported = (Passengers on direct itineraries + Passengers on connecting itineraries)

- Total Passengers Enplaned is equal to the Total Passengers Transported on direct itineraries, plus two times the Total Passengers Transported on connecting itineraries

- Passengers per flight $=[($ Passengers on direct itineraries $)+(2 *$ Passengers on connecting itineraries $)] /$ Total Flights

- Passengers per itinerary $=($ Passengers on direct itineraries + Passengers on connecting itineraries $) /$ Total Passenger Itineraries

- Average passenger itineraries per flight $=$ Total Passenger Itineraries/Total Flights

- Average passengers per direct itinerary = Passengers on direct itineraries/Total Passenger Itineraries

- Average passengers per connecting itinerary = Passengers on connecting itineraries/Total Passenger Itineraries

- Alternate definition of Passengers per flight $=(1 *$ Average passengers per direct itinerary $)+[($ Average passengers per itinerary -1$)^{*}$ Average passengers per connecting itinerary $* 2$ connections per itinerary]

- Total Passenger Trip Delays $=[\mathrm{P}($ direct itinerary disrupted $) *$ total direct passenger itineraries * Average passengers per direct itineraries * Average delay per disrupted direct itinerary $]+[\mathrm{P}$ (connecting itinerary 
disrupted) * total connecting passenger itineraries * Average passengers per connecting itineraries * Average delay per disrupted connecting itinerary ]

As can be seen in the last equation, shifts in passengers and shifts in the number of itineraries can affect the total passenger trip delay without changing the magnitude of delays or the likelihood of delays. This property is important to consider when designing metrics to incentivize consumer protection standards.

Note: in this paper, a passenger itinerary is considered to be "disrupted" by delayed flights, diverted flights, cancelled flights and by missed connections. In other research $[3,7,8]$, the term disrupted Itinerary is reserved for cancelled flights and missed connections..

\section{Passenger Itinerary (PFI) and Passenger Trip Delay (PTD) Algorithms}

Two processes are required to estimate the Passenger Trip Delays for the APTS. The first process generates an estimate of the passenger itineraries. The second process uses the itineraries and the flight performance to estimate the passenger trip delays.

\section{A. Data Sources}

Data for the analysis is derived from three publicly available government data-bases from the Bureau of Transportation Statistics [8]:

(1) The Airline Origin and Destination Survey (DB1B) Market: is a $10 \%$ sample of airline tickets from reporting carriers provided on a quarterly basis. Data includes origin, destination and other itinerary details of passengers transported. The data is provided in the form of Origin-Destination, or Origin-Hub-Destination.

(2) The Air Carrier Statistics (Form 41 Traffic) T100 data-base contains domestic non-stop segment data reported by both U.S. and foreign air carriers, including carrier, origin, destination, aircraft type, available capacity, and load factor. Data is provided on a monthly basis.

(3) The Airline On-Time Performance (AOTP) data-base contains on-time arrival data for non-stop domestic flights by major air carriers, and provides such additional items origin and destination airports, flight numbers, scheduled and actual departure and arrival times, and cancelled or diverted flight status. The data is provided on a flight-by-flight basis for each day.

\section{B. Passenger Itinerary Generation}

The Passenger Itinerary Generation algorithm generates the passenger itineraries and estimates the number of passengers on each itinerary. The DB1B provides a list of the generic itineraries flown by passengers (e.g. DCA-ATL-DEN) that is used to generate specific passenger itineraries for each day (e.g. 06/07/2007: DL 1137 DCA-ATL departing 09:00, DAL 1243 ATL-DEN departing 11:45) from the AOTP.

The DB1B also provides an estimate of the total passengers per quarter on each itinerary that is used to estimate the percentage of passengers on a given passenger itinerary. The T100, providing an estimate of the total passengers on each individual flight, is used in conjunction with the percentage of passengers on each itinerary to estimate the number of passengers on a passenger itinerary.

The Passenger Itinerariues are generated by the following 6 steps:

Step 1 Identify all the possible itineraries: The DB1B is used to generate all the O-D and O-H-D itineraries flown in a month. These itineraries identify the sequence of aiports flown by passengers with an airline (e.g. Delta Airlines: DCA- ATL, Delta Airlines : DCA-ATL-DFW). Itineraries with more than 2 segments account for $2.5 \%$ of the itineraries in 2007 and are ignored for this analysis. Only itineraries with more than 0.5 passengers per day were considered in this analysis.

Step 2 Identify the passenger itineraries: The AOTP is used to generate the individual passenger itineraries for each O-D and O-H-D itinerary in DB1B. The output is a list of O-D flights and O-H-D for each day. For example, On April $1^{\text {st }} 2007$, AOTP identifies two flight itineraries that provide service on DCA-ATL-DFW: DL-417 DCA-ATL departing at 9am and arriving at 11:00am and DL-471 ATL-DFW: departing at 12:25pm and DL-1137 DCA-ATL departing at 8pm and arriving at 9:55pm connecting to DL-1697 ATL-DFW departing at 10:40pm.

Step 3 Assign Seat Capacity and Total Passengers to each Flight: The T100 data-base is used to assign seats available and total passengers on each flight in the Flight Itineraries.

Step 4 Estimate Total Passengers for each Day on each Itinerary: For each DB1B generic itinerary (e.g. DCA-ATL-DFW) the total passengers in a quarter is multiplied by 10 to generate an estimate the total quarterly passengers on the itinerary. The total quarterly passengers are divided by 90 days to estimate the total passengers per day on each itinerary. For example, DB1B estimates that 360 passengers flew an itinerary DCA-ATL-DFW during a specified quarter (i.e. 90 days). This amounts to 4 passengers per day on this itinerary. 
Step 5 Estimate the Percentage of Passengers on each Segment of each Itinerary: Sort the itineraries that have an X-Y airport pair by airline and flight number and date. The X-Y could be in an O-D itinerary, an O-H itinerary, an O-H segment or an $\mathrm{H}-$ $\mathrm{D}$ segment on an O-H-D itinerary. Divide the passenger count on individual itineraries by the sum the total passengers that share the X-Y segment. This yields the percentage of passengers on each X-Y segment of the itinerary. For example, on April $1^{\text {st }} 2007$, the passengers travelling on DL-1137 with an itinerary DCA-ATL-DFW accounted for 3\% of the total number of passengers on that flight. Similarly passengers originating at DCA and terminating at ATL (direct itinerary) accounted for $15 \%$ of the total number of passengers on DL-1137 (see Table 2).

Step 6 Estimate Passengers on each Flight in each Flight Itinerary: The T-100 average passengers per flight (Step 3) and the Percentage of Passengers on each segment of each Itinerary (Step 5) are used to estimate the number of passengers on each flight in each itinerary. On direct itineraries 10 passengers are added to account for a biased under estimation of passengers on DB1B direct itineraries. On connecting itineraries, passengers are allocated evenly between the different itineraries up to the actual load factor from T100. Passenger counts are rounded up as follows: flights with less than 0.3 passengers are assigned 0 passengers, flights with 0.3 to 0.9 passengers are rounded up. For example, the average number of passengers on DL-1137 travelling from DCA to ATL were 122 (Step 3) and the percentage of passengers with itinerary DCA-ATL-DFW was $3 \%$. This means that out of 122, only 4 passengers are travelling on that itinerary. Similarly, the percentage of passengers with a direct DCA-ATL itinerary was $15 \%$, which means 19 passengers on DL-1137 originate at DCA and terminate at ATL.

A sample of a passenger itinerary for a day is shown in Table 2.

\begin{tabular}{|c|c|c|c|c|c|c|c|c|c|c|c|}
\hline ORIGIN & DEST & HUB & AIRLINE & DB1B PAX & FL_1_NUM & Seats & $\begin{array}{c}\text { T100 Load } \\
\text { Factor }\end{array}$ & FL_2_NUM & Seats & $\begin{array}{c}\text { T100 Load } \\
\text { Factor }\end{array}$ & $\begin{array}{c}\text { Pax Flight } \\
\text { Itin Pax }\end{array}$ \\
\hline DCA & ATL & NULL & DL & 63 & 1137 & 143 & 0.853146853 & NULL & NULL & NULL & 19 \\
\hline LGA & ATL & DCA & DL & 18 & 1961 & 134 & 0.507462686 & 1137 & 143 & 0.853146853 & 10 \\
\hline LGA & ATL & DCA & DL & 18 & 1963 & 134 & 0.507462686 & 1137 & 143 & 0.853146853 & 10 \\
\hline DCA & BHM & ATL & DL & 27 & 1137 & 143 & 0.853146853 & 574 & 146 & 0.739726027 & 8 \\
\hline DCA & CHS & ATL & DL & 16 & 1137 & 143 & 0.853146853 & 1164 & 142 & 0.866197183 & 5 \\
\hline & & & & & & & & & & & \\
\hline DCA & TPA & ATL & DL & 19 & 1137 & 143 & 0.853146853 & 836 & 201 & 0.815920398 & 6 \\
\hline
\end{tabular}

Sample Passenger Itinerary generated from DB1B, AOTP, and T100 data-bases. The "Pax Flight Itin Pax" in the last column is generated from the percentage of passengers on each itinerary and the load factor for each flight from T100. Table 2

An alternate scheme for generating passenger itineraries is described in [9]. This approach uses historical data and a discrete logit model to determine load factors based on a day-of-week and time-of-day basis

\section{Passenger Trip Delay Algorithm}

The algorithm for estimating Passenger Trip Delays uses the Passenger Itineraries described above and the Airline OnTime Performance (AOTP) data-base. The AOTP data-base contains on-time arrival data for non-stop domestic flights.

The Passenger Trip Delay Algorithm is summarized in Figure 1. For each day in the period under analysis, each passenger itinerary is processed. If the itinerary is a connecting itinerary, the algorithm follows the left branch (shaded). If the $\mathrm{O}-\mathrm{H}$ flight is cancelled, passengers are rebooked on an itinerary by the same airline from the origin to the destination. The rebooked passenger itinerary may be direct, connect through the original hub, or connect through an alternate hub. The Passenger Trip Delay for these passengers is based on the frequency of service and availability of seats. Passengers that cannot be rebooked on the day of the flight are assigned a Passenger Trip Delay of 900 minutes (i.e. 15 hours) an estimate of the delay accrued to be rebooked on a flight the following day [3, 4].

If the $\mathrm{O}-\mathrm{H}$ flight is not cancelled, the algorithm checks to see if the $\mathrm{O}-\mathrm{H}$ flight was diverted. An estimate of the delay due to the diversion is computed based roundtrip flying time and turnaround time to the closest airports with appropriate length runways. If the diverted flight returns to the Hub airport and misses the connecting flight, the passengers are rebooked from the Hub to the Destination on the same airline. The rebooked passenger itinerary is direct to the destination. The Passenger Trip Delay for these passengers is based on the Passengers that cannot be rebooked on the day of the flight are assigned a Passenger Trip Delay of 900 minutes (i.e. 15 hours) an estimate of the delay accrued to be rebooked on a flight the following day.

Next the algorithm checks the arrival time on the $\mathrm{O}-\mathrm{H}$ flight. If the $\mathrm{O}-\mathrm{H}$ flight is delayed and is less than 30 minutes from the departure of the H-D flight, the passengers are considered to have missed their connection and are rebooked from the Hub to the Destination as described above.

Next the algorithm evaluates if the H-D flight is cancelled. If this flight is cancelled, the passengers are rebooked from the Hub to the Destination. The Passenger Trip Delay for these passengers is based on the Passengers that cannot be rebooked on the day of the flight are assigned a Passenger Trip Delay of 900 minutes (i.e. 15 hours) an estimate of the delay accrued to be rebooked on a flight the following day. 


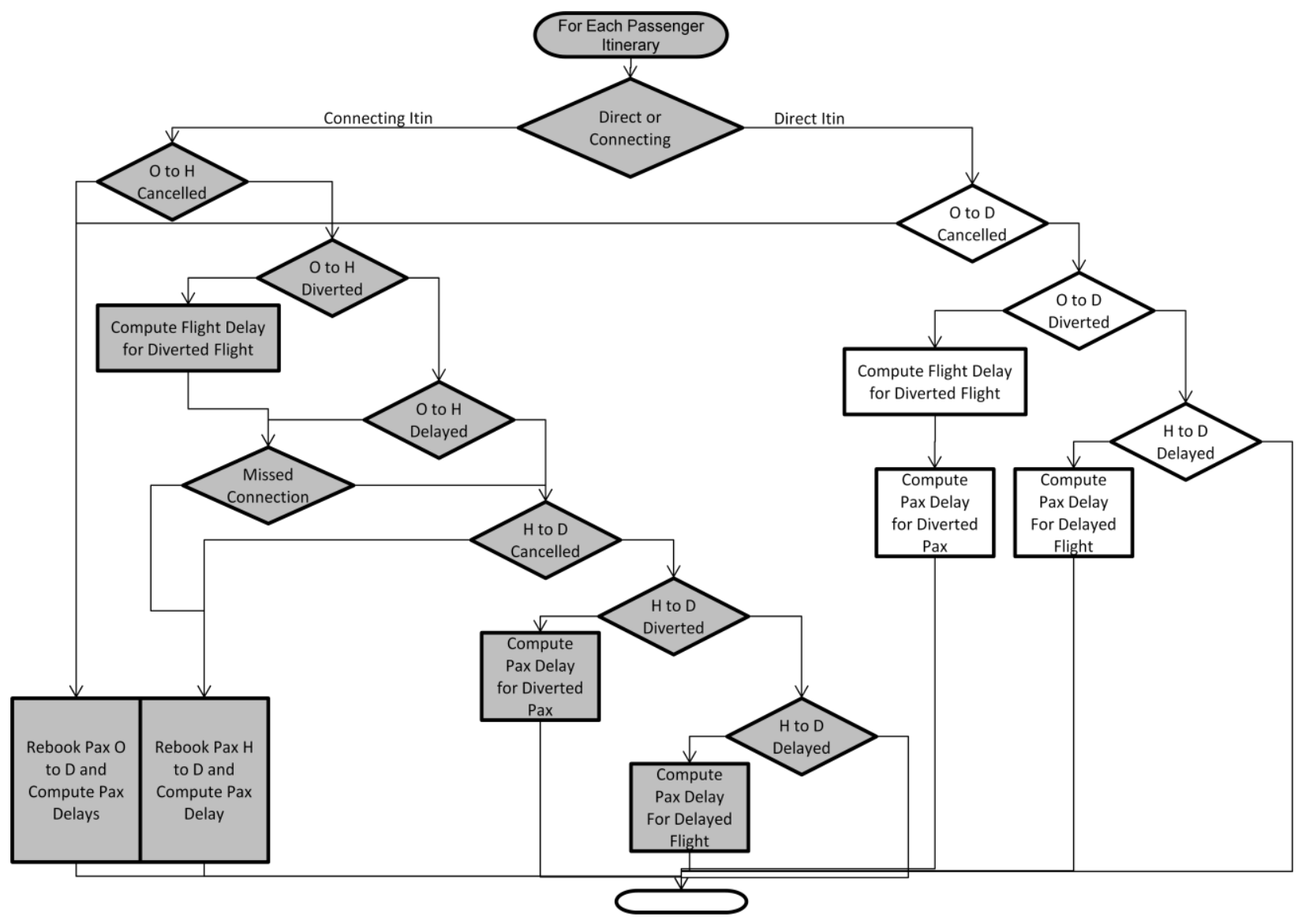

Algorithm for estimation of Passenger Trip Delays. Each itinerary is evaluated for flights on each day of under analysis. Total Passenger Trip Delays, Probabilities of Disrupted Itineraries, and Average Disrupted Itineraries Delays are computed. Figure 1

Next the algorithm checks the itinerary for a diversion on the H-D segment. If the flight is diverted, the An estimate of the delay due to the diversion is computed based on roundtrip flying time and turnaround time to the closest airports with appropriate runways.

Finally, the algorithm checks the H-D flight for delays. If the flight is more than 15 minutes late, passenger trip delay is computed.

If the passenger itinerary is a Direct Itinerary, the algorithm follows the right branch. The algorithm checks for cancelled flight, diverted flight and delayed flight, rebooking and/or assigning passenger delays as described above.

\section{Limitations and Validation}

The passenger itinerary is at best a representative estimate of the actual passenger itineraries. Historical information on passenger itineraries is considered proprietary by the airlines for competitive reasons and subject to civil liberties regulations. Absent such proprietary data, a representative estimate of itineraries based on approaches that utilize publicly available data is the best that can be realistically achieved. Sources of in the itinerary error include: (1) missing itineraries from the DB1B $10 \%$ sample, (2) aggregated quarterly DB1B passenger data, (3) the average load factor from T100 based on monthly data, and (4) the simplified algorithm that divides the number of passengers each between the available flights. The combination of these sources of error does not make distinctions between time-of-day and day-of-week.

By way of validation, a comparison of the total flights per year from AOTP to total flights per year in the passenger itineraries showed a loss of 2,133 flights per day equivalent to $10 \%$ of the total flights. In comparison to Barnhart et al [9] the total number of itineraries generated by this method is short $13 \%$. The total passengers in the Passenger Itineraries generated by this method is $6 \%$ lower than the total passengers in the T100 data but $21 \%$ higher than the total passengers in [9].

The passenger trip delay algorithm is accurate for delayed flights. Diversion delays are estimated as are the rebooking itineraries.

\section{Results}

The results of the passenger itinerary analysis provide a nuanced picture of the performance of the APTS that is hidden when the itinerary performance is not measured. This section describes the structure of the itineraries, their properties, and the resulting performance of each type of itinerary. 


\section{A. APTS Statistics}

This analysis investigated the performance of the APTS in 2007 which included 6.6 million flights were included in the network providing the passenger itineraries (see Table 3). Two and ninety-nine airports were served. On average, a given airport had direct flights to 11 airports.

During this period an estimated 579 million passengers boarded individual flights. Flights had an average 86 passengers per flight in the network. In terms of passenger itineraries, 388 million passengers booked tickets on 51 million passenger itineraries. On each flight, there was an average of 6.9 itineraries. There was an average of 7.6 passengers per itinerary.

In terms of the breakdown of direct and connecting itineraries, there were approximately 7 times more connecting itineraries than direct itineraries. Thirteen percent of the available passenger itineraries were direct itineraries, $87 \%$ of the available passenger itineraries were connecting itineraries. There were also, on average approximately 7 passengers on each passenger itinerary (30 passengers per direct itineraries and 4 per connecting itineraries). The 1:7 ratio of direct to connecting passengers along with the 7:1 ratio of direct passengers per itinerary versus connecting passengers per itinerary resulted in an equal portion of passengers on each type of itinerary. Fifty one percent of passenger trips were transported on direct itineraries, $49 \%$ of the passengers were transported on connecting itineraries. Further a typical flight will have approximately 30 passengers on direct itineraries and 56 passengers on connecting itineraries.

\begin{tabular}{|c|c|c|c|}
\hline & Total & $\begin{array}{c}\text { Direct } \\
\text { Itineraries }\end{array}$ & $\begin{array}{l}\text { Connecting } \\
\text { Itineraries }\end{array}$ \\
\hline Flights & $6,660,444$ & & \\
\hline Airports Served & 299 & & \\
\hline $\begin{array}{l}\text { Average Number of Airports Served from an Origin by } \\
\text { a Flight }\end{array}$ & 11 & & \\
\hline Total Passengers Enplaned & $579,159,038$ & & \\
\hline Average Passengers per Flight (Network) & 86 & 30 & 56 \\
\hline Number of Passenger Itineraries & $51,088,619$ & $12.8 \%$ & $87.2 \%$ \\
\hline Average Itineraries on a Flight & 7.6 & & \\
\hline Average Passengers per Itinerary & 7.6 & 30.2 & 4.3 \\
\hline $\begin{array}{l}\text { Passengers on Direct Itineraries + Passengers on } \\
\text { Connecting Itineraries }\end{array}$ & $388,127,327$ & $51.0 \%$ & $49.0 \%$ \\
\hline
\end{tabular}

The structure of the APTS in 2007. 51million itineraries transported 388 million passengers.

Table 3.

\section{B. ATPS Flight Performance}

The year 2007 was characterized by one of the worst flight performance records in recent history. An unprecedented number of flights were scheduled $(7,326,836)$ resulting in over-scheduling leading to congestion and delays when capacity was reduced (e.g. due to weather). Twenty three percent of the flights were delayed, $2.2 \%$ cancelled, and $0.23 \%$ diverted flights.

\section{Passenger Itinerary Performance Statistics}

Fifty-one million passenger itineraries were flown in 2007 (see Table 4). Thirty two percent of the total passenger itineraries experienced disruptions. Twenty seven percent of the itineraries with delayed flights, $2.3 \%$ were itineraries with cancelled flights, $2 \%$ were itineraries with missed connections, and $2 \%$ were itineraries with diverted flights.

\begin{tabular}{|l|r|r|}
\hline Passenger Itineraries & All Itineraries \\
\hline All Itineraries & $51,088,619$ & \\
\hline On-Time Itineraries & $35,160,279$ & $68.8 \%$ \\
\hline Disrupted Itineraries & $15,916,884$ & $31.2 \%$ \\
\hline Delayed Flights & $13,639,520$ & $26.7 \%$ \\
\hline Cancelled Flights & $1,161,906$ & $2.3 \%$ \\
\hline Missed Connections & $1,036,722$ & $2.0 \%$ \\
\hline Diverted Flights & 90,192 & $0.2 \%$ \\
\hline
\end{tabular}

Breakdown of Passenger Itinerary statistics by type of itinerary disruption.

Table 4

Of the 51 million itineraries, $12.8 \%$ were direct itineraries, while $87.2 \%$ were connecting itineraries (see Table 5). Connecting itineraries were 6 times more likely to be disrupted than direct itineraries. Of the $32.2 \%$ itineraries that were disrupted, $4.4 \%$ were direct itineraries and $27.9 \%$ were connecting itineraries. Connecting itineraries (27\%) were equally 
likely to experience disruptions due to a delayed flight as direct itineraries (24.4\%). Connecting itineraries (2.4\%) were 1.8 times more likely be disrupted due to a cancelled flight than direct itineraries $(0.5 \%)$. Connecting and direct itineraries were equally likely to experience a disruption due a diversion than direct itineraries $(0.1 \%)$.

\begin{tabular}{|l|r|r|r|r|}
\hline Passenger Itineraries & \multicolumn{2}{|c|}{ Direct Itineraries } & \multicolumn{2}{c|}{ Connecting Itineraries } \\
\hline All Itineraries & $6,548,426$ & $12.8 \%$ & $44,540,193$ & $87.2 \%$ \\
\hline On-Time Itineraries & $4,850,394$ & $74.1 \%$ & $30,309,885$ & $68.1 \%$ \\
\hline Disrupted Itineraries & $1,686,576$ & $25.8 \%$ & $14,230,308$ & $31.9 \%$ \\
\hline Delayed Flights & $1,595,349$ & $24.4 \%$ & $12,044,171$ & $27.0 \%$ \\
\hline Cancelled Flights & 88,821 & $1.4 \%$ & $1,073,085$ & $2.4 \%$ \\
\hline Missed Connections & $\mathrm{N} / \mathrm{A}$ & & $1,036,722$ & $2.3 \%$ \\
\hline Diverted Flights & 13,862 & $0.2 \%$ & 76,330 & $0.2 \%$ \\
\hline
\end{tabular}

Breakdown of disrupted itineraries by itinerary type (direct and connecting) and by type of disruption.

Table 5

\section{Average Passengers per Itineraries}

On average, 7.6 passengers were flown on each itinerary (see Table 6). On direct itineraries, an average of 30 passengers were flown on each itinerary, and an average of 4 passengers on connecting itineraries. This ratio (7:1) is roughly the inverse of the ratio of the number of direct and connecting itineraries.

The passengers per itinerary were not symmetric with respect to type of itinerary or type of itinerary disruption.

Disrupted itineraries had approximately 1 less passenger per itinerary than on-time passengers, an indication that itineraries intentionally disrupted by airlines for tactical reasons, tend to minimize the impact to passengers. This trend only applies to direct itineraries (not connecting itineraries).

A comparison of itinerary types by type of disruption, shows that itineraries disrupted by delayed flights, diverted flights, or missed connections had the same number of passengers per itinerary as the average for disrupted itineraries. Passengers on direct itineraries that were disrupted by cancelled flights dropped from 29 to 3 passengers per itinerary. This is a strong indication that itineraries intentionally disrupted by airlines for tactical reasons, tend to minimize the impact to passengers. The complexity of connecting itineraries may prohibit managing effect of disruptions on passengers.

\begin{tabular}{|l|c|c|c|}
\hline $\begin{array}{l}\text { Passengers per } \\
\text { Itinerary }\end{array}$ & $\begin{array}{c}\text { All } \\
\text { Itineraries }\end{array}$ & $\begin{array}{c}\text { Direct } \\
\text { Itineraries }\end{array}$ & $\begin{array}{c}\text { Connecting } \\
\text { Itineraries }\end{array}$ \\
\hline All Passengers & 7.60 & 29.96 & 4.28 \\
\hline On-Time & 7.87 & 30.38 & 4.27 \\
\hline Disrupted & 7.00 & 28.97 & 4.30 \\
\hline Delayed Flights & 7.19 & 30.17 & 4.15 \\
\hline Cancelled Flights & 6.90 & 3.12 & 5.51 \\
\hline Missed Connections & 4.31 & & 4.77 \\
\hline Diverted Flights & 8.31 & 32.56 & 3.90 \\
\hline
\end{tabular}

Passengers per itinerary by type of itinerary and by type of disruption. Approximately 7 times more passengers were transported on direct itineraries than on connecting itineraries. Direct itineraries had fewer passengers per itinerary on disrupted itineraries than connecting itineraries. This effect was driven primarily by direct itineraries disrupted by canceled flights which had only 3 passengers per itinerary (compared to 30). This is an indication of tendency by airlines to manage disruptions to minimize the effect on passengers. Their ability to do so may be limited only to direct itineraries.

Table 6

\section{E. Average Passenger Trip Delay}

The average trip delay across all passengers was 21 minutes (see Table 7), however passengers on disrupted itineraries experienced an average 71 minutes trip delay. For passengers on itineraries disrupted by delayed flights, the average delay was 41 minutes. For passengers on itineraries disrupted by cancelled flights and or missed connections, the average trip delay was in excess of 5 hours. 


\begin{tabular}{|l|c|}
\hline & $\begin{array}{c}\text { Average Passenger Trip Delay } \\
\text { (mins) }\end{array}$ \\
\hline All Passengers & 21 \\
\hline On-Time & 0 \\
\hline Disrupted & 73 \\
\hline Delayed Flights & 41 \\
\hline Cancelled Flights & 324 \\
\hline Missed Connections & 346 \\
\hline Diverted Flights & 257 \\
\hline
\end{tabular}

Breakdown of average Passenger Trip Delay by type of itinerary disruption.

Table 7

The breakdown of passenger trip delays by type of itinerary and by type of itinerary disruption is shown in Table 8 . Passengers on direct itineraries experienced lower average trip delays than those passengers on connecting itineraries. The average trip delays for disrupted passengers on direct itineraries was 53 minutes, compared with 73 minutes for connecting itineraries.

The average trip delay for itineraries disrupted by delayed flights was the same for direct and connecting itineraries (40 minutes). However, the average trip delay for passengers on itineraries disrupted by cancelled flights was 57 minutes lower for direct itineraries (4 hours and 39 minutes), compared with connecting itineraries (5 hours and 36 minutes).

\begin{tabular}{|l|c|c|}
\hline & $\begin{array}{c}\text { Average Passenger } \\
\text { Trip Delay (mins) for } \\
\text { Direct Itineraries }\end{array}$ & $\begin{array}{c}\text { Average Passenger Trip } \\
\text { Delay (mins) for } \\
\text { Connecting Itineraries }\end{array}$ \\
\hline All Passengers & 14 & 29 \\
\hline On-Time & 0 & 0 \\
\hline Disrupted & 53 & 73 \\
\hline Delayed Flights & 41 & 40 \\
\hline Cancelled Flights & 279 & 336 \\
\hline Missed Connections & $\mathrm{N} / \mathrm{A}$ & 313 \\
\hline Diverted Flights & 251 & 265 \\
\hline
\end{tabular}

Breakdown of average passenger trip delays by itinerary type and by type of itinerary disruption. Passengers on connecting itineraries disrupted by cancelled flights experience the highest average trip delays.

Table 8

\section{F. Passenger Trip Delay Performance Statistics}

In 2007, 32\% of the 16.5 million passenger itineraries experienced a disruption (see Table 9). These disruptions affected 112 million passengers. The total impact of these disruptions was the equivalent of 15,841 years.

\begin{tabular}{|l|r|r|}
\hline Total Passenger Trip Delays & $8,326,178,732$ (mins) \\
\cline { 1 - 2 } Disrupted Passengers & 15,841 (years) & \\
\hline Disrupted Passenger Itineraries & $111,846,936$ & $19.3 \%$ \\
\hline
\end{tabular}

Total Passenger Trip Delays.

Table 9

The breakdown of passenger trip delays by type of disrupted itinerary is shown in Figure 10. Passenger trip delays generated by itineraries disrupted by delayed flights accounted for $48 \%$ of the total passenger trip delays. Fifty percent of the total trip delays were the result of itineraries disrupted by cancelled flights $(31 \%)$ and itineraries disrupted by missed connections $(19 \%)$. 


\begin{tabular}{|l|r|r|}
\hline & \multicolumn{2}{|l|}{$\begin{array}{l}\text { Total Passenger Trip Delays } \\
\text { (minutes) }\end{array}$} \\
\hline All Passengers & $8,326,178,732$ & \\
\hline On-Time & 0 & \\
\hline Disrupted & $8,326,178,732$ & \\
\hline Delayed Flights & $3,988,861,145$ & $47.9 \%$ \\
\hline Cancelled Flights & $2,597,897,766$ & $31.2 \%$ \\
\hline Missed Connections & $1,546,921,374$ & $18.6 \%$ \\
\hline Diverted Flights & $192,498,447$ & $2.3 \%$ \\
\hline
\end{tabular}

Breakdown of total passenger trip delay by type of itinerary disruption. Itineraries disrupted by cancelled flights and missed connections account for $50 \%$ of the total passenger trip delay.

Table 10

The breakdown of total passenger trip delay by type of itinerary and type of disruption is summarized in Table11. Thirty three percent of the total passenger trip delay occurred on direct itineraries, $67 \%$ on connecting itineraries.

For direct itineraries, itineraries disrupted by delayed flights accounted for $73 \%$ of the total delay. Cancelled flights accounted for only $22 \%$ of the total delay. In contrast, for connecting itineraries, delayed flights (35.5\%) and cancelled flights (35\%) accounted for $71 \%$ of the delay. Missed connections also had a significant contribution of $28 \%$.

\begin{tabular}{|l|c|c|c|c|}
\hline Total Passenger Trip & \multicolumn{2}{|c|}{$\begin{array}{c}\text { Total Passenger Trip Delay: } \\
\text { Delay }\end{array}$} & \multicolumn{2}{c|}{$\begin{array}{c}\text { Total Passenger Trip Delay: } \\
\text { Connecting Itineraries }\end{array}$} \\
\hline Disrupted & $2,720,439,346$ & $32.7 \%$ & $5,605,739,386$ & $67.3 \%$ \\
\hline Delayed Flights & $1,996,600,327$ & $73.4 \%$ & $1,992,260,818$ & $35.5 \%$ \\
\hline Cancelled Flights & $610,214,072$ & $22.4 \%$ & $1,987,683,694$ & $35.5 \%$ \\
\hline Missed Connections & $\mathrm{N} / \mathrm{A}$ & & $1,546,921,374$ & $27.6 \%$ \\
\hline Diverted Flights & $113,624,947$ & $4.2 \%$ & $78,873,500$ & $1.4 \%$ \\
\hline
\end{tabular}

Breakdown of total passenger trip delays by type of itinerary and type of itinerary disruption. Passengers on connecting itineraries disrupted by cancelled flights generated disproportionately highest total trip delays.

Table 11

\section{Conclusions}

One of the key measures of the performance of the Airline Passenger Transportation System (APTS) is the on-time reliability of passenger trips and the magnitude of passenger trip delays. Because the APTS service is provided through combinations of flights that are used to create passenger itineraries, the reliability of the system is determined by the reliability of the passenger itineraries.

\section{A. The Value of Passenger Itinerary Viewpoint}

The analysis of the APTS, from the point of view of the passenger itineraries, establishes an important distinction between the disruptions to itineraries due to delayed or cancelled flights and disruptions to itineraries due to cancelled flights or missed connections. In the former, performance is determined by the on-time reliability of flights and the magnitude of flight delays. In the latter, performance is determined by the response to the itinerary disruption by rebooking passengers on alternate itineraries. The frequency of service to destinations of cancelled flights along with the number of available seats determines the robustness in response to itinerary disruptions that require rebooking passengers (not just the reliability of the flights).

\section{B. Limitation of the "One-size Fits All" ATSP Performance Metrics}

Flight specific flight performance metrics are not representative of passenger trip delay for two reasons. First, as pointed out by Bratu \& Barnhart ${ }^{1}$, flight delays are poor proxies for passenger trip delays as they do not account for the delays accrued by cancelled flights, and missed connections. Second, flight performance metrics also fail to account for the structure of the schedule. Hub-and-spoke carriers that rely on connecting passengers are subject to performance metrics related to how well the hubbing operation is coordinated. Excessive flight delays coordinated across a bank operation results in improved passenger trip performance. However, these carriers are very sensitive to cancelled flights. A small increase in cancelled flights can result in a large increase in passenger trip delay. In contrast, point-to-point carriers, that experience lower cancellation rates, are more sensitive to flight delays.

\section{Impact of ATC Modernization Initiatives on Passenger Trip Performance}


Initiatives to modernize Air Traffic Control to create additional capacity will yield (at least initially) direct reductions in flight delays. This will improve the performance of passenger itineraries disrupted by flight delays. Increased capacity will have a secondary effect of possibly reducing cancelled flights (at least those not cancelled due to mechanical or "tactical" reasons). Although this will improve the performance of passenger itineraries for cancelled flights, the effect of the rebooking of passengers is dependent on the schedule and flight load factors.

\section{Passenger Bill of Rights}

A Passenger Bill of Rights is governmental rule-making that sets service standards for airline passengers. The European Union (EU) established a common set of rules related to compensation and assistance of passengers in the event of cancellations, long delays and denied boarding. In short, the EU's Air Passenger Rights mandates compensation for passengers in the event of denies boarding based on distance of flight. In the event of long delays passengers must be provided services, meals, hotel accommodation or the option for reimbursement. Financial compensation for a cancelled flight is due unless the airline has informed passengers of the flights' cancellation 14 days prior to the flight, or if the passengers have been rerouted close to their original travel times. Airlines are exempt from compensation should the cancellation be due to extraordinary circumstances.

The U.S. Passenger Bill of Rights includes only provisions for denied boarding and for extended delays (>2 hours) on the tarmac. This bill of rights does not mandate compensation for delays and cancellations. Based on the passenger itinerary analysis, the absence of cancellations and missed connections in the bill are significant omissions, as these factors are significant contributors to individual total passenger trip delays.

\section{Acknowledgments}

Thank you to Peg Young, (BTS), Thea Graham, Dan Murphy, Joe Post, Dave Chin, Kimberly Noonan (FAA), Natalia Alexandrov, Michael Madson, Rosa Oseguerra-Lohr, Maria Consiglio (NASA), Poornima Balakrishna, Ben Levy (Sensis Corp.), Chris Brinton (Mosaic ATM), Gregg Wollard (MWAA), Taryn Lewis, Norm Fujisaki, Terry Thompson, Jerome Lard, Arash Yousefi (Metron Aviation), John Shortle, John Ferguson, David Schaar, Mariecl Medina, Vivek Kumar, Yimin Zhang, and Akshay Belle. Funding for this research was provided by the FAA DTFAWA-08-F-GMU, NASA NRA NNH06ZEA001N, and internal GMU Research Foundation Funds.

\section{References}

${ }^{1}$ GRA, Inc. (2004) Economic values for FAA investment and regulatory decisions-a guide. Report prepared for FAA Office of Aviation Policy and Plans, Washington D.C.

${ }^{2}$ NEXTOR (2010) Total Delay Impact Study: A Comprehensive Assessment of the Costs and Impacts of Flight Delay in the United States. Report prepared for FAA Air Traffic organization - Strategy and Performance Business Unit Washington D.C.

${ }^{3}$ Bratu, S., Barnhart, C. (2005). An Analysis of Passenger Delays Using Flight Operations and Passenger Booking Data.

Journal of Transportation and Statistics, Number 1, Volume13, 1-27

${ }^{4}$ Wang, D., L. Sherry (2006) Passenger Trip Metric for Air Transportation. In proceedings of the 2nd International Conference on Research in Air Transportation - ICRAT 2006.

${ }^{5}$ Sherry, Lance, D. Wang (2007) Air Travel Consumer Protection: Metric for Passenger On-Time Performance.

Transportation Research Record, Transportation Research Board of the National Academies, Volume 2007, pages 22-27.

${ }^{6}$ Sherry, L. \& Calderon-Meza, G. (2008) Passenger Trip Delays in the U.S. Airline Transportation System. In Proceedings International Conference on Research in Air Transportation (ICRAT-2008), Fairfax, VA, 2008

${ }^{7}$ Zhu, Y. (2007) Evaluating Airline Delays: The Role of Airline Networks, Schedules and Passenger Demands. Masters

Thesis, MIT, Cambridge, Massachusetts. Advisor: Cynthia Barnhart

${ }^{8}$ Bureau of Transportation and Statistics (2009). Airline On-Time Performance Data, Form 41 Traffic T-100 Domestic Segment Data, DB1_B Coupon Data. Available: http://www.transtats.bts.gov. 2010 Integrated Communications Navigation and Surveillance (ICNS) Conference, May 11-13, 2010

${ }^{9}$ Barnhart, C., D. Fearing, and V. Vaze (submitted 2010) Modeling Passenger Travel and Delays in the National Air Transportation System. Submitted to Operations Research, August 3, 2010. 